\title{
Estudos de adaptação do Millon Clinical Multiaxial Inventory-III para avaliação de aspectos psicopatológicos da personalidade no Brasil
}

\author{
Adaptation Studies of the Millon Clinical Multiaxial Inventory-III \\ to assess psychopatological aspects of personality in Brazil
}

Hannia Roberta Rodrigues Paiva da Rocha', Heloísa Karmelina Carvalho de Sousa', João Carlos Alchieri', Eleni de Araújo Sales', João Carlos Nascimento de Alencar ${ }^{1}$

\section{RESUMO}

Objetivos: Traduzir e adaptar para uso no Brasil o Millon Clinical Multiaxial Inventory-III (MCMI-III), verificando as modificações envolvidas em relação à escala original. Método: 538 participantes responderam ao MCMI-III, ao Questionário de Saúde Geral de Goldberg e a um questionário sociodemográfico especificamente elaborado para esta pesquisa. Os sujeitos foram, então, divididos entre grupo clínico e não clínico, e seus padrões de respostas foram comparados considerando-se as diferentes variáveis sociodemográficas. Resultados: Os

\section{Palavras-chave}

Psicopatologia, transtornos de personalidade, avaliação.

\section{Keywords}

Psychopathology, personality disorders, evaluation. resultados demonstram diferenças estatisticamente significativas nos padrões de respostas para a maioria das escalas quando comparados os grupos clínico e não clínico. Conclusão: O estudo ratifica a sensibilidade de discriminação entre os diferentes grupos, apresentando indícios da validade do instrumento quanto ao preceito teórico.

\section{ABSTRACT}

Objectives: To translate and adapt the Millon Clinical Multiaxial Inventory-III (MCMI-III) for use in Brazil, testing the changes performed in the original scale. Method: 538 participants answered the MCMI-III, the Goldberg General Health Assessment and a social demographical questionnaire specifically designed to this research. Then, respondents were divided into clinical and non-clinical groups, and their answers' patterns were compared considering different social demographic characteristics. Results: Significant statistical differences in answer patterns were found in most scales when clinical and non clinical groups were compared. Conclusion: This study confirms the ability of the MCMI-III to discriminate different groups, consistent with the instrument validity based his theoretical foundations.

\section{INTRODUÇÃO}

O Millon Clinical Multiaxial Inventory (MCMI) foi um dos instrumentos desenvolvidos por Theodore Millon para operacionalizar sua teoria e tem como foco pessoas em tratamento clíni- co em psicologia ou psiquiatria'. Desde sua criação, em 1977, o instrumento passou por três atualizações ${ }^{2}$ para que pudesse guardar estreita relação com as mudanças sociais que se operavam em cada época e se refletiam no aperfeiçoamento do Diagnostic and Statistical Manual of Mental Disorders (DSM).

1 Universidade Federal do Rio Grande do Norte (UFRN). 
O DSM configura-se como um manual diagnóstico e estatístico adotado pela APA, que se associa com a Classificação de Transtornos Mentais e de Comportamento (CID), da Organização Mundial de Saúde (OMS). Seu sistema classificatório é multiaxial, organizado de forma a comportar 16 classes diagnósticas diferentes, que recebem códigos numéricos específicos e são agrupados em cinco grandes eixos. Os aspectos avaliados pelo MCMI-III são aqueles que no DSM-IV-TR se encontram nos dois primeiros eixos: o eixo I, que descreve os transtornos clínicos propriamente ditos, e o eixo II, correspondente ao retardo mental, bem como aos transtornos de personalidade 3 . Como todo sistema de classificação, o DSM-IV-TR não é um sistema infalível e não pode fornecer automaticamente um diagnóstico, portanto é recomendável cautela em sua utilização.

Dessa forma, a primeira versão do inventário, cujas normas foram estabelecidas a partir de estudo com mais de 1.500 pacientes psiquiátricos, visava avaliar personalidade e síndromes clínicas ${ }^{4}$. A segunda edição foi publicada em 1987, consoante às mudanças sociais ocorridas ao logo dos dez anos posteriores à publicação da primeira versão, adequando-se ao DSM-III-R. O MCMI-III, por sua vez, foi desenvolvido em 1994, seguindo as atualizações do DSM-IV4,5. Vale salientar que a atualização do DSM-IV para o DSM-IV-TR envolveu apenas revisão textual, não havendo modificação nos termos diagnósticos ${ }^{3}$.

A versão mais atual do MCMI é o terceiro instrumento para avaliação da personalidade mais utilizado no contexto internacional, ficando atrás apenas do Rorschach e do Minnesota Multiphasic Personality Inventory-ll, em termos de produção científica. Essa versão conta com 175 itens de resposta verdadeiro ou falso, que podem ser respondidos no formato papel e lápis ou por meio de um aplicativo informatizado. O tempo de administração pode variar entre 20 e 40 minutos de acordo com o nível de leitura dos indivíduos, sendo apropriado para uso em adultos (acima dos 18 anos de idade) que estejam em processo de avaliação psicológica ou em acompanhamento de saúde mental. A consistência do teste está entre 0,57 e 0,95,6.

O MCMI, em todas as suas versões, distingue-se de outros inventários principalmente pela sua fundamentação teórica, estrutura multiaxial, esquema de validação, bem como pela brevidade com que se faz a sua aplicação e a análise e interpretação de seus dados. Cada edição do inventário procurou manter um número total de itens suficientemente pequenos para motivar a sua utilização nos mais variados tipos de diagnóstico e de tratamento, porém grande o bastante para proporcionar a análise de um amplo quadro de comportamentos clinicamente relevantes. O teste, então, é consideravelmente menor do que outros instrumentos internacionais utilizados para avaliação de aspectos psicopatológicos da personalidade 5 .

O MCMI-III possui quatro escalas de verificação do instrumento: Validade, Divulgação, Desejabilidade e Valoriza- ção Negativa, que podem ser analisadas separadamente ou inter-relacionadas, mas as escalas de Validade e Divulgação podem invalidar o teste 6 . A escala de Validade ( $V$ ) consiste em três declarações improváveis, e se duas delas forem respondidas positivamente, o teste é invalidado. Portanto, essa escala é a que primeiro deve ser analisada. A escala de Divulgação $(X)$ propõe-se a identificar a disposição para admitir sintomas e problemas que o sujeito possui, bem como a franqueza com que ele se expõe. A escala de Desejabilidade $(Y)$ verifica a tendência do sujeito em responder aos itens de forma favorável, ou seja, a disposição em realizar o teste. A escala de Valorização Negativa (Z), por sua vez, afere a possibilidade de que o sujeito responda ao teste acentuando ou exagerando problemas e sintomas que possui ou, ainda, citando problemas que de fato não apresenta ${ }^{5,7}$.

As demais escalas do instrumento referem-se a cada tipo de desordem de personalidade identificada na teoria de Millon e dividem-se em: escalas de padrões clínicos de personalidade (Esquizoide, Evitativa, Depressiva, Dependente, Histriônica, Narcisista, Antissocial, Agressiva/Sádica, Compulsiva, Negativista e Autodestrutiva/Masoquista), escalas de transtornos de personalidade severa (Esquizotípica, Paranoide e Borderline), escalas de síndromes clínicas moderadas (Transtorno de Ansiedade, Transtorno de Somatização, Transtorno Bipolar, Transtorno Distímico, Dependência Alcoólica, Dependência de Drogas e Transtorno do Estresse Pós-Traumático) e escalas de síndromes clínicas graves (Desordem de Pensamento, Depressão Maior e Transtorno Delirante) $)^{5}$.

Beck et al. ${ }^{8}$ enfatizam um problema envolvido no diagnóstico de transtornos da personalidade: raramente os problemas da personalidade constituem a principal demanda de um paciente que busca tratamento. Tais pacientes têm dificuldades em lidar com outras pessoas independentemente de sua própria conduta. Na maior parte das vezes, esse paciente se sente vitimizado pelo mundo e pela sociedade. Alguns têm noção dos elementos que envolvem seus problemas, mas não apresentam a consciência dos aspectos da personalidade na mudança de seu comportamento; daí, uma das maiores dificuldades em diagnosticar transtornos da personalidade.

Estudos que utilizam o MCMI-III como instrumento de avaliação psicológica sugerem que o teste é um dos mais adequados para avaliação de certos transtornos. Marcus et al. ${ }^{9}$ postulam que transtornos da personalidade sejam abordados como se manifestando em uma dimensão contínua e não como um diagnóstico categórico. O MCMI, portanto, se configuraria como um instrumento ideal para mensurar tais transtornos.

Internacionalmente, o MCMI-III vem sendo utilizado em pesquisas associadas à psicologia forense, principalmente para a avaliação de população carcerária ${ }^{10}$, de abusadores sexuais ${ }^{11}$ e de usuários de álcool e outras drogas ${ }^{12-15}$. No Brasil, 
nenhuma das versões do instrumento foi adaptada anteriormente. Carvalho ${ }^{16}$ utilizou a tradução preliminar do MCMI-III, realizada para este estudo, como parâmetro para a validação de um instrumento de avaliação de aspectos psicopatológicos da personalidade baseado no modelo teórico de Millon.

O presente estudo, então, tem como objetivo a adaptação do Millon Clinical Multiaxial Inventory-IIl para o Brasil, verificando as modificações envolvidas em relação à escala original.

\section{MÉTODO}

\section{Participantes}

Participaram deste estudo 538 respondentes, dos quais 294 $(54,5 \%)$ do sexo feminino e $243(45,2 \%)$ do sexo masculino (um participante não forneceu essa informação), divididos em dois grupos - grupo clínico e grupo não clínico. Para diferenciar os grupos, dada a inexistência no momento de um critério de padrão-ouro (diagnósticos psiquiátricos) em virtude da ausência de diagnósticos precisos e de fontes confiáveis, optou-se pela distinção baseada na autopercepção e autodescrição do respondente quanto à presença de indicadores como: o histórico ou antecedentes de psicopatologias, atendimentos e tratamentos psicológicos e/ou psiquiátricos com ou sem uso de psicofarmacoterapia, além da referência a abuso de álcool e/ou outras drogas, não se fazendo distinção entre transtornos mentais menores, maiores ou adicções, sendo a verificação dessa categorização esperada na análise dos padrões de respostas dos participantes. Muito embora essa distinção possa ser considerada tênue, pareceu aos autores uma forma preliminarmente viável de verificar possíveis distinções entre os grupos antes de se recorrer aos critérios específicos na sequência de estudos ulteriores que já se delineiam abordando especificamente alguns transtornos identificados pelo MCMI-III.

O grupo clínico, portanto, foi composto por pessoas que referiram durante a resposta ao instrumento algum tipo de necessidade de cuidado ambulatorial em psicologia ou psiquiatria (198 sujeitos, o que corresponde a 36,8\% da amostra). Os sujeitos do grupo clínico foram cooptados nos Serviços de Saúde Mental da cidade de Natal, Rio Grande do Norte, quais sejam: as clínicas-escolas das instituições de ensino superior com graduação em psicologia no estado (Universidade Federal do Rio Grande do Norte - UFRN - e Universidade Potiguar - UnP) e os Centros de Atenção Psicossocial (CAPS), nas modalidades de atendimento em saúde mental geral e para usuários de álcool e drogas; além dessas instituições da capital do estado, foram coletados dados no CAPS de Currais Novos, município do interior do RN. Dentro das clínicas-escolas, foram abordadas as pessoas que aguardavam atendimento para triagem, psicoterapia ou avaliação psicológica. Nos CAPS, os sujeitos foram indicados por membros da equipe profissional dentre aqueles que se voluntariaram para participação na pesquisa e puderam, durante o tempo necessário para a aplicação dos instrumentos, ausentar-se das atividades da casa sem nenhum prejuízo ao sujeito ou grupo de atividade.

O grupo não clínico, por sua vez, foi composto por pessoas sem referência a alguma história ou necessidade de cuidado psicológico e ou psiquiátrico (340 sujeitos, perfazendo 63,2\% da amostra), cooptadas entre os acompanhantes dos pacientes dos mesmos serviços citados no grupo clínico, além de indivíduos que se submeteram à avaliação para seleção profissional ou vocacional, estudantes universitários e pessoas da população geral que aceitaram participar do estudo.

Os dois grupos foram compostos por adultos com idades de 18 a 85 anos, segundo indicações do instrumento original, não havendo seletividade específica para os níveis de escolaridade, embora esse dado fosse identificado no questionário sociodemográfico para utilização estatística posterior.

Como critérios de exclusão, foram considerados a idade dos participantes (menos de 18 anos e mais de 85), o número de respostas omissas e/ou marcadas em duplicidade (que não deve ser superior a 11, segundo orientações do instrumento original) e, no caso das pessoas que compuseram o grupo clínico, o tempo em que estavam em tratamento. Considerou-se que a evolução do paciente que passou por mais de duas semanas de acompanhamento terapêutico poderia interferir nas respostas dadas às sentenças do MCMI-III, influenciando, assim, seu resultado, optando-se, portanto, pela sua exclusão dos grupos. Após a tabulação dos dados, verificou-se que 17 dos 538 sujeitos que responderam ao instrumento apresentaram número de respostas omissas ou marcadas duplamente, sendo, então, excluídos da amostra analisada para o presente estudo.

\section{Instrumentos}

Além do MCMI-III, todos os sujeitos da amostra responderam a um questionário sociodemográfico, especificamente elaborado para os fins desta pesquisa. Os usuários dos CAPS responderam, ainda, ao Questionário de Saúde Geral (QSG), com o objetivo de verificar a possibilidade de distúrbios menores do ponto de vista psiquiátrico. Além do QSG também foram utilizados em subgrupos da amostra o teste de Zulliger, o Inventário Millon de Estilos de Personalidade (MIPS), o Millon Behavioral Medical Diagnostic (MBMD), este último também em fase de adaptação.

\section{Procedimentos}

Após aprovação pelo Comitê de Ética do Hospital Universitário Onofre Lopes (com o número 324/09), foram realizados os contatos com as instituições nas quais se realizaria a coleta de dados para autorização da pesquisa nesses locais.

Os participantes, em ambos os grupos, eram abordados e recebiam uma explicação sobre seus direitos como partici- 
pantes da pesquisa e, após o esclarecimento de eventual dúvida e a manifestação do interesse em participar do estudo, assinavam o Termo de Consentimento Livre e Esclarecido e eram convidados a se dirigir a local mais reservado para administração do instrumento. Por causa da pouca experiência em leitura da maioria dos sujeitos abordados nas instituições de cuidado para saúde mental, os instrumentos foram aplicados individualmente, com leitura e marcação das respostas feitas pelo aplicador. A parcela do grupo não clínico constituída por universitários recebeu as mesmas orientações dos sujeitos anteriores. No entanto, com esse grupo foi possível realizar administração coletiva do instrumento.

Concomitantemente à coleta de dados, foi desenvolvido um banco de dados e construído um aplicativo de avaliação e correção informatizado (no formato de planilha eletrônica) para ser empregado nos procedimentos de análise inicial dos resultados das escalas, uma vez que estas, dadas as características do instrumento, são compostas de uma variedade de pontuações e valores para os itens, dificultando seu uso no formato tradicional em papel. Portanto, a elaboração de tal aplicativo foi compatível com a necessidade do presente estudo, permitindo a utilização imediata dos resultados após digitação dos dados.

\section{RESULTADOS}

Dos sujeitos que tiveram os dados analisados, 284 (54,5\%) participantes eram do sexo feminino e 236 eram do sexo masculino (um foi omisso quanto a essa questão), com 105 dos respondentes com escolaridade fundamental, $212 \mathrm{com}$ ensino médio e 196 com ensino superior (8 sujeitos não informaram sua escolaridade). Compuseram o grupo clínico, então, 193 sujeitos e o grupo não clínico, 328.

Os resultados demonstram diferenças significativas entre as médias dos fatores para o grupo clínico e não clínico nas escalas de verificação: X (Divulgação), Y (Desejabilidade Social) e Z (Valorização Negativa), todas com $p<0,001$. Quanto aos resultados das escalas de padrões clínicos de personalidade, evidenciaram-se diferenças nas escalas 1 (Esquizoide), escala 2B (Depressivo), escala 3 (Dependente), escala 6A (Antissocial) e escala 8B (Masoquista), com significância de $p<$ 0,001 e na escala 6B (Sádico/Agressivo), com $p=0,001$. Em relação às escalas de transtornos de personalidade severos, apresentaram diferenças significativas a escala $S$ (Esquizotípico) $(p=0,001)$ e a escala C (Borderline) $(p<0,001)$. As escalas de síndromes clínicas moderadas H (Somatoforme), D (Distimia), T (Dependência de Drogas), B (Dependência de Álcool) e R (Transtorno do Estresse Pós-Traumático) apresentaram significância de $p<0,001$, enquanto a escala A (Ansiedade) apresentou $p=0,01$. De igual forma, observaram-se diferenças entre as médias nas escalas de síndromes clínicas severas - escala SS (Transtorno do Pensamento), escala CC
(Depressão Maior) e escala PP (Transtorno Delirante), todas $\operatorname{com} \mathrm{p}<0,001$.

Nesse sentido, as médias de pontuações brutas para os grupos clínico e não clínico nas diferentes escalas de transtornos de personalidade se distribuíram de acordo com a tabela 1 .

Em relação ao conjunto de escalas de síndromes clínicas, os resultados são os apresentados na tabela 2.

Tabela 1. Médias das pontuações brutas nas escalas de transtornos de personalidade (grupo clínico x não clínico)

\begin{tabular}{|c|c|c|c|}
\hline Transtornos de personalidade & Grupo & Média & Desvio-padrão \\
\hline \multirow[t]{2}{*}{ Esquizoide* } & Clínico & 10,7 & 5,2 \\
\hline & Não clínico & 6 & 4,4 \\
\hline \multirow[t]{2}{*}{ Depressivo* } & Clínico & 13,2 & 6,6 \\
\hline & Não clínico & 6,1 & 5,5 \\
\hline \multirow[t]{2}{*}{ Dependente $^{*}$} & Clínico & 12,8 & 6,2 \\
\hline & Não clínico & 6,7 & 5,2 \\
\hline \multirow[t]{2}{*}{ Antissocial* } & Clínico & 11,2 & 5,7 \\
\hline & Não clínico & 6,2 & 3,6 \\
\hline \multirow[t]{2}{*}{ Sádico-agressivo** } & Clínico & 13,2 & 6 \\
\hline & Não clínico & 8,5 & 5 \\
\hline \multirow[t]{2}{*}{ Masoquista* } & Clínico & 10,1 & 6 \\
\hline & Não clínico & 4,1 & 4,5 \\
\hline \multirow[t]{2}{*}{ Esquizotípico** } & Clínico & 13 & 7 \\
\hline & Não clínico & 6,3 & 5,9 \\
\hline \multirow[t]{2}{*}{ Borderline* } & Clínico & 12,5 & 6,6 \\
\hline & Não clínico & 5,7 & 4,9 \\
\hline \multirow[t]{2}{*}{ Ansiedade*** } & Clínico & 12,1 & 5,2 \\
\hline & Não clínico & 6,4 & 4,6 \\
\hline \multirow[t]{2}{*}{ Somatoforme* } & Clínico & 8,2 & 5,1 \\
\hline & Não clínico & 3,8 & 4,2 \\
\hline \multirow[t]{2}{*}{ Distímico* } & Clínico & 10,6 & 6,1 \\
\hline & Não clínico & 3,9 & 4,7 \\
\hline
\end{tabular}

${ }^{*} p=0,000 ;{ }^{* *} p=0,001 ;{ }^{* * *} p=0,010$

Tabela 2. Médias das pontuações brutas nas escalas de síndromes clínicas (grupo clínico x não clínico)

\begin{tabular}{llll}
\hline Sindromes clínicas & Grupo & Média & Desvio-padrão \\
\hline Dependência de álcool* $^{*}$ & Clínico & 8,3 & 5,5 \\
& Não clínico & 3,6 & 2,8 \\
Dependência de drogas* $^{*}$ & Clínico & 8,2 & 6,2 \\
& Não clínico & 2,9 & 2 \\
Transtorno do estresse pós-traumático* & Clínico & 11,3 & 6,4 \\
& Não clínico & 4,5 & 4,9 \\
Transtorno do pensamento* & Clínico & 12,8 & 6,1 \\
& Não clínico & 6,7 & 5 \\
Depressão maior* & Clínico & 9,9 & 7 \\
& Não clínico & 3,7 & 5 \\
Transtorno delirante* & Clínico & 8,8 & 4,7 \\
& Não clínico & 4,7 & 3,7 \\
\hline
\end{tabular}

${ }^{*} p=0,000$ 


\section{DISCUSSÃO}

Mesmo sendo o grupo clínico amplo e sem diferenciação diagnóstica, foi possível evidenciar distinções altamente significativas para a maior parte das escalas, especialmente entre as de síndromes clínicas. A evidência de diferenças entre os fatores e o sexo é concordante com a literatura, especificamente se tomados os transtornos de personalidade como o Antissocial $(p=0,000)$, Masoquista/Autodestrutivo $(p=0,001)$ e as Síndromes Clínicas de Ansiedade $(p=0,010)$, Dependência de Álcool $(p=0,000)$ e Dependência de Drogas $(p=0,000)$.

Tabela 3. Médias das escalas cujos padrões de respostas apresentaram diferenças estatisticamente significativas segundo o sexo

\begin{tabular}{llllll}
\hline & Sexo & N & Média & $\begin{array}{l}\text { Desvio- } \\
\text { padrão }\end{array}$ & $\begin{array}{l}\text { Médiade } \\
\text { erro-padrão }\end{array}$ \\
\hline Antissocial* $^{*}$ & Feminino & 284 & 6,62 & 4,065 &, 241 \\
Masoquista** $^{*}$ & Masculino & 236 & 9,76 & 5,653 &, 368 \\
& Feminino & 284 & 5,68 & 5,626 &, 334 \\
Ansiedade*** $^{*}$ & Masculino & 236 & 7,12 & 6,029 &, 392 \\
Dependência de álcool* $^{*}$ & Feminino & 284 & 8,25 & 5,330 &, 316 \\
& Masculino & 236 & 8,86 & 5,858 &, 381 \\
Dependinino & 284 & 3,95 & 3,300 &, 196 \\
& Masculino & 236 & 7,05 & 5,318 &, 346 \\
& Feminino & 284 & 3,36 & 3,121 &, 185 \\
& Masculino & 236 & 6,66 & 5,791 &, 377 \\
\hline
\end{tabular}

${ }^{*} p=0,000 ;{ }^{* *} p=0,001 ; * * * p=0,010$.

Os estudos preliminares da adaptação brasileira do MCMI-III, então, ratificam a sensibilidade de discriminação entre diferentes grupos subjacente à teoria de Millon, o que é corroborado por estudo de Brandão e Vasco ${ }^{17}$, realizado com o MCMI-II, segundo o qual grupos de sujeitos com diferentes diagnósticos (esquizofrenia, psicose e toxicodependência) responderam de forma significativamente diferente às escalas Dependente, Antissocial e Compulsiva ( $p<0,05)$, Passivo-Agressivo, Negativista, Esquizotípico, Borderline, Ansiedade, Somatoforme, Bipolar, Distímico, Dependência de Álcool, Dependência de Drogas, Transtorno do Pensamento e Depressão Maior $(p<0,01)$.

Rossi et al. ${ }^{18}$ também enfatizaram o poder discriminativo do MCMI-III ao encontrarem diferenças significativas nos padrões de respostas de diferentes grupos quando comparados por sexo $(p<0,001$ para todas as escalas, exceto Agressivo, Compulsivo, Bipolar e Transtorno Dissociativo) na adaptação holandesa do instrumento, com médias maiores para as mulheres nas escalas de Ansiedade, Somatoforme, Distimia, Transtorno do Estresse Pós-Traumático, Transtorno do Pensamento e Depressão Maior.
Vanem et al..$^{19}$, ao compararem adictos e pessoas que não realizavam uso de substância, identificaram diferenças entre os dois grupos nas respostas para as escalas de Dependência de Drogas $(M=94,77, D P=15,11)$, Antissocial $(M=82,95, D P$ $=12,86)$ e Depressão $(M=74,33, \mathrm{DP}=26,66)$.

Outro estudo vem corroborar os dados analisados na presente discussão. Sinha e Watson ${ }^{20}$ sugerem, mediante pesquisa feita com 157 estudantes de psicologia, que o MCMI-III indica um padrão distintivo de relação entre fatores psicossociais e transtornos de personalidade específicos. As informações obtidas em tal estudo explicitam uma porcentagem relativamente grande de variância em praticamente todos os transtornos de personalidade.

\section{CONCLUSÃO}

É possível, mesmo de forma preliminar e com base na autorreferência por parte dos respondentes, verificar que a versão brasileira do instrumento tem apresentado médias significativamente distintas quando considerados grupos clínico e não clínico, caracterizando um dos indícios de validade quanto ao preceito teórico. Cabe salientar que, mesmo não havendo uma diferenciação nosológica ou mesmo sintomática que respalde a constituição do grupo clínico, a sensibilidade do instrumento foi capaz de apresentar um aspecto diferenciador e, em consequência, possivelmente promissor na evolução dos estudos de validade. Com a continuidade da coleta de dados e a ampliação da amostra, estudos seguem sendo desenvolvidos na busca de identificação e caracterização de aspectos complementares nas diferenças entre sexo, idade e escolaridade dos sujeitos. Busca-se, dessa forma, elementos que possam amparar a verificação da estrutura fatorial do MCMI-III, esperando-se que esta corrobore o preconizado por Millon quanto às características psicopatológicas segundo a composição de grupos critérios, além da verificação da validade e da especificidade do instrumento.

\section{AGRADECIMENTOS}

À Coordenação de Aperfeiçoamento de Pessoal de Nível Superior e ao Conselho Nacional de Desenvolvimento Científico e Tecnológico pelo apoio financeiro.

A todos os acadêmicos de Psicologia participantes do Projeto de Extensão de Avaliação Psicológica à Comunidade e do Projeto de Pesquisa para Adaptação do MCMI-III para o Brasil.

\section{REFERÊNCIAS}

1. Ellis A, Abrams M. Personality theories: critical perspective. In: Ellis A, Abrems M. Personality and psychometric tests: objective personality measures. Califórnia: Sage Publications, Inc; 2009, p. 67-70 
2. Millon T, Davis R, Millon C. MCMI-III: Inventário Multiaxial de Millon - III - Manual. TEZ Ediciones; 2007

3. Heim A, Westen D. Essentials of personality disorders. In: Oldham JM, Skodol AE, Bender DS. Personality disorders: recent history and the DSM system. Arlington: American Psychiatric Publishing Inc; 2009, p. 3-13.

4. Millon T, Millon CM. The Millon inventories: clinical and personality assessment. In: Millon T. History, theory and validation of MCMI. New York: The Guildford Press; 1997, p. 23-40.

5. Craig RJ. Essentials of Millon inventories assessment. In: Strack S. Essentials of MCMI-III assessment. 3a ed. Nova York: John Wiley \& Sons; 2008, p. 1-55.

6. Millon T, Davis R, Millon C, Grossman S. The Millon Clinical Multiaxial Inventory - III. 3a ed. Minneapolis: Pearson Assessment; 2006.

7. Millon T, Rogers D, Millon C, Grossman S. The Millon Clinical Multiaxial Inventory-III. 3a ed. [with new norms and updated scoring]. Disponível em://www.millon.net/instruments/ MCMI_III.htm. Acesso em: 4 out 2010.

8. Beck AT, Freeman A, Davis DD, et al. Terapia cognitiva dos transtornos de personalidade. $2^{2}$ ed. Porto Alegre: Artmed; 2007.

9. Marcus DK, Ruscio J, Lilienfeld SO, Hughes KT. Converging evidence for the latent structure of antisocial personality disorder: consistency of taxometric and latent class analyses. Crim Justic Behav. 2008;35(3):284-93.

10. Duque AF, Neves PG. Automutilação em meio prisional: avaliação das perturbações da personalidade. Psic Saúde Doenças. 2004;5(2):215-27.
11. Pechorro PS, Poiares C, Vieira RX. Caracterização psicológica de uma amostra forense de abusadores sexuais. Aná Psicológica. 2008;21(4):615-23.

12. Herrero J. Alteraciones de la personalidad asociadas a las conductas adictivas: influencia de la duración del consumo y sus implicaciones. Psykhe. 2004;(13)1:91-100.

13. González MCT, Riveros MMP, Uribe Jl, Luna SM. Validación de la prueba Young Adult Alcohol Problems Screening Teste, YAAPST, en un grupo de estudiantes universitarios de la Pontificia Universidad Javeriana de Bogotá. Univ Psychol. 2006;1(5):175-90.

14. Hérran OF, Ardila MF, Barba DM. Consumo problemático de alcohol en Bucaramanga, CoIombia: diseño y validación de una prueba. Biomédica. 2007;(28)1:25-37.

15. Pérez CL, Lara CV. Asertividad, resistencia a la présion de grupo y consumo de alcohol en universitarios. Acta Colomb Psicol. 2008;(11)1:155-62.

16. Carvalho LF. Construção e validação do Inventário Dimensional de Transtornos da Personalidade [dissertação]. Itatiba: Universidade São Franscisco; 2008.

17. Brandão A, Vasco AB. Discrepâncias do eu: diferenças estruturais entre as populações clínica e não clínica avaliadas com o MCMI-II. Psicólogos. 2008;3(1):22-52.

18. Rossi G, Van der Ark LA, Sloore H. Factor analysis of the Dutch-language version of the MCMI-III. J Personal Assess. 2007;88(2):144-57.

19. Vanem PC, Krog D, Hartmann E. Assessment of substance abusers on the MCMI-III and the Rorschach. Scand J Psychol. 2008;49(1):83-91.

20. Sinha BK, Watson DC. Psychosocial predictors of personality disorder traits in a non-clinical sample. Pers Individ Dif. 1997;22(4):527-37. 